\title{
WEB APPLICATIONS DASHBOARD AS A TOOL FOR DATA VISUALIZATION USING CLOUD DATA SERVERS
}

\author{
B.Harika \\ Dept of Computer Science \\ \& Engineering \\ NS Raju Institute of \\ Technology
}

\author{
B.Prem Kumar \\ Dept of Computer Science \\ \& Engineering \\ NS Raju Institute of \\ Technology
}

\author{
Ch. Deepak \\ Dept of Computer Science \\ $\&$ Engineering \\ NS Raju Institute of \\ Technology
}

\author{
R.Sandhya \\ Dept of Computer \\ Science \& Engineering \\ NS Raju Institute of \\ Technology
}

\author{
G. Srinivasarao \\ Assistant Professor \\ Department of Computer Science \& Engineering \\ NS Raju Institute of Technology
}

\begin{abstract}
Working spot of a person that oversees information investigation and information handling can affect one individual or an entire group. The instruments that can mama e their work simpler, diminish the work hours and at last decrease the quantity of individuals required in the group are dashboards of web applications. With the assistance of dashboards one can oversee, break down and outwardly screen their key measurements identified with their business or to a person. There is no all-inclusive dashboard to suit each type of business. Similarly, there is no widespread interface that can be proclaimed as the lone appropriate answer for all organizations, in this manner various dashboards are being created in various interfaces. People or groups are the clients of dashboards. There are various dashboard layouts for site interfaces that can assist engineers with making their own dashboards for open information all the more effectively and rapidly. The point of the paper is to break down and assess different dashboard layouts as indicated by the characterized measures to exhibit which format is the most proper for a specific area and field of work. It is normal that the outcomes will assist engineers with the decision of a dashboard layout for their new ventures. This paper gives essential rules with respect to responsive website composition and a portion of the systems that can be utilized to assemble a responsive website composition page. It likewise momentarily portrays the CSS Grid. Besides, it'll dissect the graduated alumni dashboard format made inside four unique frameworks and CSS Grid. They were investigated with five given rules the 5 second standard, the reversed pyramid, information perception, toning it down would be ideal and intuitive components. The outcomes would
\end{abstract}

underscore the formats made inside bootstrap and Semantic I since they were not assessed by any basis with a score under 4 out of 5 .

Keywords: Alumni dashboard, data visualization, Responsive web application.

\section{INTRODUCTION}

The enormous measure of information has prompted the requirement for a proficient method of checking. Generally, business-related data is composed inside tables that have regularly been misty and tedious. What's more refreshing bookkeeping pages and following individual information set aside time that could be utilized to tackle business issues. This is the place where dashboards become an integral factor. With dashboards, information following is neither tedious nor obscure.

Veronica S. Smith, in her work "Information Dashboard as an Evaluation and Research Communication Tool", expresses that dashboards are visual portrayals that track, break down, and present the main data expected to accomplish explicit objectives, and are as such recorded on a solitary screen. The accommodation of dashboards is that the information is continually refreshed and gives modern knowledge into the condition of business [1].

There are various dashboard formats accessible on the Internet that are made inside Frameworks or with the assistance of CSS Grid that make Responsive Web Design. Such formats are not difficult to introduce and alter, contingent upon the desires and needs of end clients. 


\section{RESPONSIVE WEB DESIGN}

Responsive website architecture implies that site pages are having the option to adjust to various screen widths, so a responsive website architecture is a way for a developer to plan a site page to coordinate with the various widths of the gadgets from which it was seen. This method of making a page guarantees that the substance of the page is effectively seen and that the information on the website page is lucid, paying little mind to the element of the gadget from which it was seen [2].

With the expanding creation of gadgets of various widths, there was a need to foster responsive website pages. Over the most recent couple of years, portable deals have outperformed work area deals, and in this manner Google has focused on versatile cordial pages in indexed lists. The term Responsive Design was initially utilized by a website specialist Ethan Marcotte. An article from 2010 approached A List Apart talks about how website architecture ought not be stale and that new gadgets should be joined by fitting plan. He concluded that making new pages for each kind of gadget would not be a decent arrangement, yet that there ought to be a plan that would adjust with its adaptability and ease to various screens [3].

In his work Responsive Web Design: Present and Future, Muhammad Rizwan Pasha makes reference to four essential standards of adjusting site pages to various screen widths.

The main key guideline applies to a Fluid/Flexible Grid. With regards to site pages, a framework is a bunch of lines and sections and is utilized to put together the presence of individual components inside a page. The adaptable network communicates values in rates by the accompanying recipe:

(target/setting) $\times 100=$ wanted worth $\%$ (result adjusted to 0 decimal spot) Another essential guideline applies to adaptable media like recordings and pictures. The goal of this guideline is to try not to appoint a fixed width to the predefined media, however to put them in a holder to which the relative worth that is communicated as a rate is added.

The third major rule applies to media questions containing individual CSS decides that apply to width, stature, shading, goal, and so forth There are two kinds of pixels in this unique situation: gadget pixels and CSS pixels. CSS pixels change by zooming in or out. For instance, on the off chance that a client broadens the page to $150 \%$, CSS pixels will increment by half in width and stature, yet the gadget pixels don't change by zooming in or out of individual substance. A media inquiry comprises of at least one articulations for a given screen width that might be valid or bogus. On the off chance that the media question is valid, the terms given for the fitting screen width are applied alongside the remainder of the CSS components recorded.
The fourth crucial rule identifies with dynamic substance. Dynamic substance alludes to content that changes dependent on the conduct, interests, or inclinations of an individual client [4].

Responsive Web Design framework's Regardless of the designer's information, making an adjustable site from the beginning is an exceptionally difficult and confounded errand. That is the reason engineers will in general pick a predefined workplace or the purported Responsive Web Design Frameworks.

A Framework is characterized as a bundle comprising of the construction of documents and envelopes of normalized code (HTML, CSS, JS, and so on) Making a plan through a picked Framework is straightforward. The designer ought to be acquainted with the documentation of the specific Framework inside which the site is assembled. The objective of the Framework is to save time and empower some sort of comprehensiveness. It gives designers a notable code structure so they don't squander energy on composing the construction of the page, and it lessens a large part of the functioning hours [5].

Positively, there are hindrances that accompany this method of making a responsive site. Inside most systems, by far most of potential situations that can happen when planning a site are anticipated. In this way, notwithstanding the stuff for a fashioner to make an adjustable site, there is frequently an excess of code. Another downside is that the adaptability to establish is decreased on the grounds that the workplace accompanies predefined styles and norms. So assuming an individual needs to change something inside the predefined records, it will take a great deal of time, and there is likewise the chance of accidentally changing the construction of the predefined documents [6]. There are different kinds of responsive website composition structures, and those that will be shrouded in more detail in this paper are: Foundation, Bootstrap, Materialize and Semantic UI. Establishment is a responsive frontend system that comprises of HTML, CSS, and JavaScript documents to help engineers fabricate a responsive website page. Establishment was made by ZURB in 2008 and was only used to assemble sites for their clients. Afterward, they joined what they thought was a decent establishment and an extraordinary alleviation to any designer, and introduced the Foundation to the general population without precedent for 2011. From the earliest starting point, even today, the Foundation is for nothing. The way that the Foundation has gotten perhaps the most popular structures is proven by the way that it is utilized by a huge number of architects and designers around the world. The current form of this structure that is presently modern is Foundation 6. This refreshed form permits you to make quality improved pages for a wide range of screens. Inside the Foundation there will be there is likewise the Business Foundation that offers proficient assistance, 
advising, instruments and preparing for singular organization laborers who decide to utilize this system when planning and creating pages for their customers. The documentation and backing they give shows the amount they care about their clients. With the most recent update, they have worked on the documentation and added a Getting started archive that offers fast knowledge into how the Foundation functions. They have made a Foundation Forum for all clients to post likely inquiries and offer their experience [7]. A responsive website architecture system called Bootstrap was planned and carried out by Twitter fashioners and designers in 2011. Prior to being known as responsive website architecture structure, Bootstrap was at first known as Twitter Blueprint (2010). In the primary months of its presence, Twitter coordinated an undertaking called Hack Week which was upheld by different engineers who contributed significantly to the further improvement of these stages. Along these lines, engineers of these stages had the option to choose in which course they needed to foster the style of both Twitter and Bootstrap. Bootstrap is at present kept up with by a group of designers on GitHub. Since its delivery to people in general, Bootstrap has had more than twenty more modest releases and three huge ones. Interestingly, a bigger delivery called Bootstrap 2 has had the option to make responsive site pages. The current delivery is Bootstrap 4, which incorporates the transition to Sass and CSS flexboxes. Bootstrap engineers urge the advancement local area to utilize the more current CSS highlights and make new innovation accessible through present day programs [8].

Appear is a responsive website architecture system made and planned by Google. This structure was made to coordinate exemplary plan standards with development and innovation. When constructing this plan language, Google is engineers were directed by research on paper and ink, and with the assistance of material reality, made alleged material plan. Appear intends to give a special client experience across all Google items. Through different components like pictures, typography and tones, engineers place accentuation on visual solace and put center around explicit substance. Emerge comes in two distinct structures. Contingent upon information and necessities, the designer picks among Materialize and Sass. Appear contains a normalized rendition of the code that incorporates both the full and limited variants of CSS and JavaScript documents. The Sass alternative incorporates SCSS records that require a Sass compiler, yet with this choice, the engineer can pick what segments to incorporate while making the site [9].

Semantic UI is likewise one of responsive frontend structures that is upheld by Leaner Style Sheets and j query. Semantic UI is diverse on the grounds that rather than shortenings, which are generally utilized by different systems, it utilizes genuine words that take after normal English. Its uniqueness is additionally apparent using five classes to characterize conduct inside the workspace, specifically: UI Elements, UI Collection, UI View, UI Module and UI Behavior [10].

\section{B. Cascading Style Sheet Grid}

Falling Style Sheet Grid, usually known as CSS Grid, is a method of getting sorted out content on pages. CSS Grid is a two-dimensional framework that at the same time oversees sections and lines. CSS was constantly used to set the design of components on a site page, yet it wasn't generally commonsense or easy to understand. It is therefore that structures have been made. Nonetheless, there are additionally a few drawbacks inside systems in regards to the exhibition and size of the libraries that should be incorporated while chipping away at a task inside a specific structure. Subsequent to finding out about the CSS Grid, there was a worry that responsive website architecture structures would stand out forever, yet that is positively not the situation. Flexboxes are likewise extremely supportive when constructing an adaptable site, yet they are more expected for one dimensional substance the executives [11]. CSS Grid is explicitly intended to address the appearance and situating of content in a two-dimensional framework across various gadget widths.

As referenced beforehand, before the CSS Grid, different strategies were utilized to characterize components utilizing CSS. As innovation has advanced, so has the requirement for another method of characterizing components. Since this sort of characterizing components (inline-block, drift, and so forth) isn't fixed, if the page were seen at various sizes, these components would occupy the room contingent upon the size of the gadget from which they were seen, in opposition to what the fashioner planned. This is the reason most originators and engineers pick a fixed presentation so the components can't be extended or covered in a given space. CSS Grid furnishes originators and engineers with an instrument that partitions accessible space into sections and pushes and cleverly resizes components across website pages and applications. For cell phones and tablets, tailor site pages and applications to the vertical and scene direction of the gadget. CSS Grid joins the presence of a network with media inquiries so the creator doesn't need to reorder the components inside the .html document, however characterizes the area of the component inside the CSS record to accomplish the ideal design in the two directions [12]. Making a responsive website page with CSS Grid is simple. The engineer constructs the lattice and sorts out the substance inside it, applying CSS rules to the parent component, which turns into a network holder, and to the offspring of that component. At the absolute starting point of record creation, we characterize the parent component, which has become a matrix compartment, with show: network. Segments and lines are characterized utilizing matrix layout sections and framework format pushes, and are remembered for network segment and lattice line components. The request in which the components are written in a HTML archive is 
superfluous, in light of the fact that a similar request can be totally adjusted by a couple of lines of code in CSS, which is the reason CSS Grid is an extremely integral asset [13].

\section{DATA DASHBOARDS}

Dashboards are adaptable for the assorted necessities of individual organizations or foundations. The dashboard works by interfacing with an assortment of data sets, connections, administrations and APIs, and at last the on dashboard client sees everything as tables, graphs, and alternate methods of showing data. The incredible benefit of a dashboard is that it accumulates information from various sources into one spot and gives you a powerful outline of them. In the previous method of working together, a large part of the employee is time was spent examining information. Presently they don it invest energy on information investigating since dashboards offer constant information observing so representatives can manage other business issues.

There is no widespread dashboard to suit each sort of business. That is why the significant piece of altering your dashboard is to pick the key exhibition pointers [14]. A quality dashboard addresses significant business questions and gives a simple toutilize examination of those answers. Planning a proficient dashboard is enormously helped with the standard of an upset pyramid. The rule of the altered pyramid shows that the main business markers or individual exercises that the client needs to screen are set at the earliest reference point. Along these lines, the main markers are in any case and accordingly draw in the important consideration structure the client. The remainder of the pyramid is loaded up with subtleties comprising of more broad data for a more profound comprehension of the information [15]. Notwithstanding, consider how individuals of various capacities utilize the dashboard. In this way, it is ideal to have one dashboard for explicit clients who are in sure capacities in the business, in light of the fact that not all clients are keen on a similar business-related data. Along these lines, it is suggested that various dashboards are made for the designated bunch so everybody can rapidly and effectively access the information they need to proceed with their quality work. All together for clients of dashboards to know initially what bearing their business is going, picking the right tones is significant. It has become commonly acknowledged that green shows something positive, safe, and energizes individuals, while red demonstrates risk and demonstrates alert. Legitimate utilization of these two tones can give a fast understanding in the condition of the business. Additionally, different tones ought to add to the dashboard is concordance, instead of occupy clients. A significant factor in building a viable dashboard is the measure of information that is apparent on the dashboard. The normal individual can hold somewhere in the range of five and nine information in their momentary memory. Whatever surpasses a given number requires more investigation and reflection with respect to the individual [16].
A significant factor in making a dashboard is the way you outwardly present your information. That implies that the creator should realize what is the most ideal approach to picture each kind of information. The motivation behind information perception is to unmistakably pass on the upsides of the information in diagrams. All together for a solitary representation to really pass on and show singular information, track down the most ideal manner by which that information can be shown, instead of utilizing singular types of information show basically in light of the fact that they are "cool". When utilizing structures that current information which are not insightfully picked, it confounds the clients and in light of the data introduced they arrive at resolutions more troublesome. Line graphs are utilized to give clients understanding into the patterns of various classes that occur over a similar period. Pie outlines are valuable to show what the entire is comprised of and how much space does every thing possess. Underline in pie outlines that it is ideal to utilize something like six or less various upsides of the entire to give a decent quality information see, so the contrasts between the qualities are more noticeable [17].

These were only a portion of the approaches to envision information. Be that as it may, not all things are apparently. The main part is that an assortment of information can be embedded into the dashboard, which, aside from magnificence apparently, advertisements on usefulness in a literal sense. The objective of the dashboard is to give clients a moment understanding into the condition of the business, not to think extra. Show the setting of the data gave. Generally, the current information incorporates significant information from the past period, contingent upon the sort of information being shown. All together for the dashboard to be pretty much as proficient as could really be expected, furnish the end client with a viable perspective on the information on which his business depends [18].
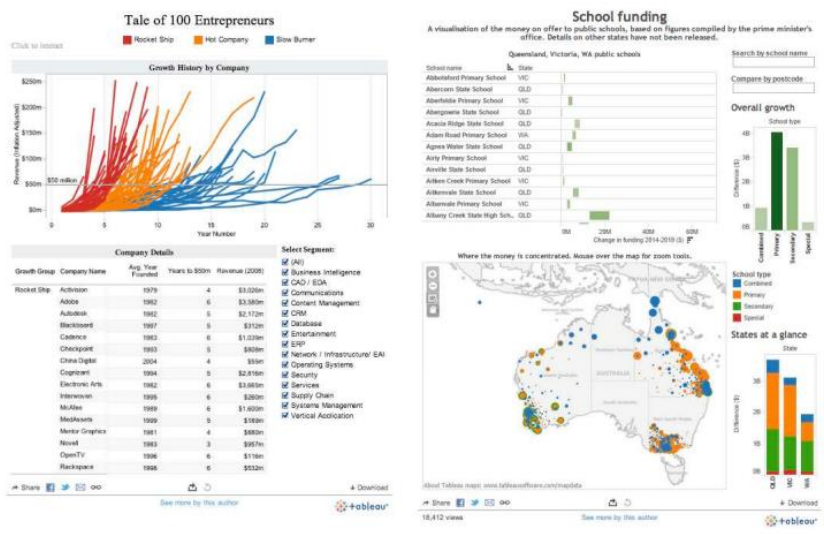

Fig. 1. Data Processing and Visualisation Tools 


\section{International Journal of Engineering Applied Sciences and Technology, 2021 \\ Vol. 6, Issue 2, ISSN No. 2455-2143, Pages 71-76 \\ Published Online June 2021 in IJEAST (http://www.ijeast.com)}

\section{THE RESEARCH}

The research includes finding and installing dashboard templates that are created within mentioned responsive web design frameworks and with the help of CSS Grid. Subsequently, individual dashboard templates were evaluated by defined criteria.

\section{A. Criteria for e Valuating dashboards}

Comparative Analysis on Data Visualization for Operations Dashboard [19] set out certain criteria by which dashboards are being valued. The first criterion involves understanding and refers to the correct definition of the most relevant data. The second and third criteria are related to the visual aspect of the dashboard. They mostly deal with interactive and dynamic elements. The fourth criterion discusses the importance of using the correct visual representations for each type of data. The fifth criterion is about improving the ability to remember certain data that is of utmost importance to the business or college, here we are working alumni dashboard.
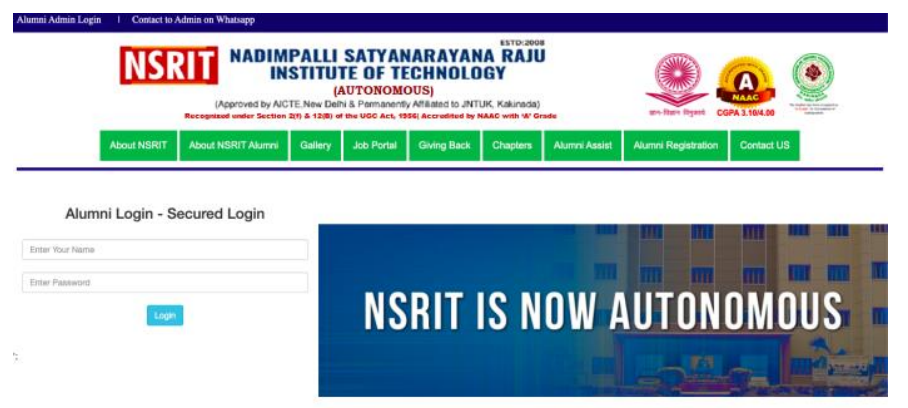

Fig 2: Welcome Screen Can student login

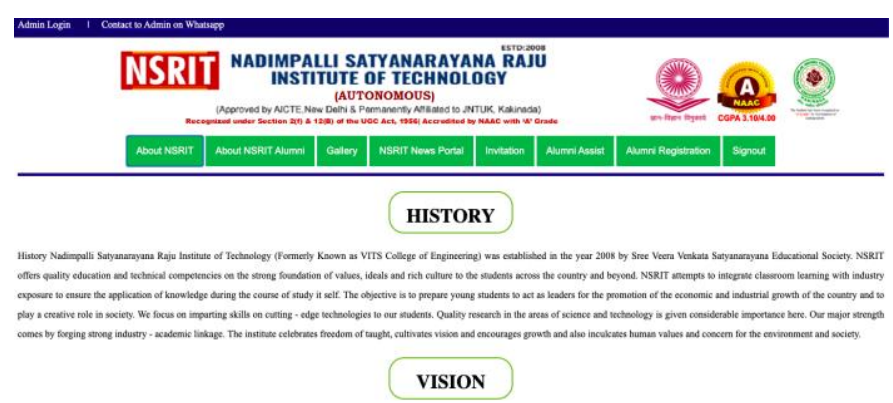

Fig 3: After Login this home screen will display
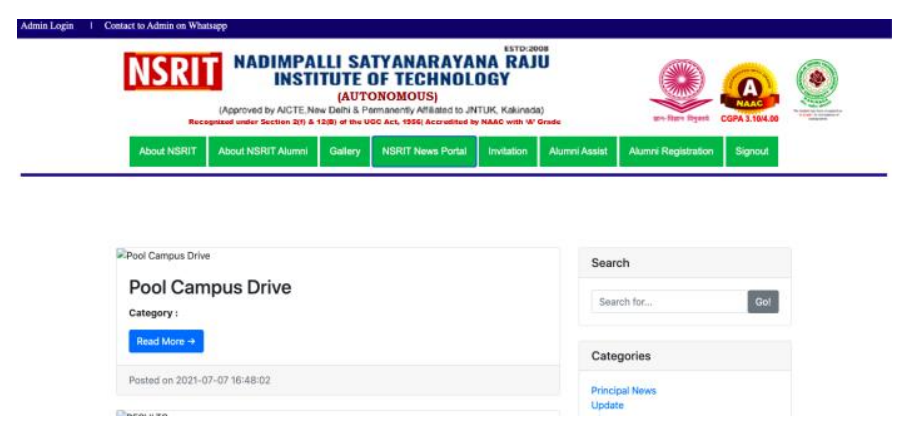

Fig 4: New Portal which can view by Alumni Students

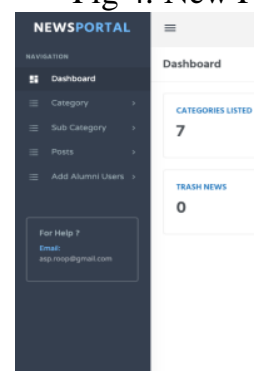

Fig 4: Admin Portal to Update News, Notifications

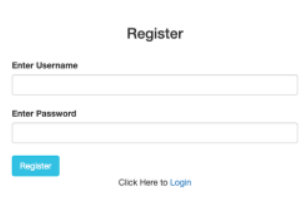

Fig 5: Register Students in Portal by Alumni Admin

\section{CONCLUSION}

Dashboards have greatly facilitated business around the world. With the help of dashboards, all business-relevant information is in a single place. The fact that dashboards save time goes in favor of employees because they no longer having to manually summarize and track data, but can monitor it in real time. Different types of business require a different view of the hierarchy among the data, which is where dashboard templates come into play. The dashboard template can be easily installed and customized by the developer who organizes and presents the data by the user is requirements.

When choosing an effective dashboard template, it is important to determine if there are different ways of visualizing the data that is needed for the business, then it is important to set the desired hierarchy among the data and thus identify the most important ones. It is equally important to briefly summarize the data so that the dashboard users will at first glance get specific information about the current state of business, not to further confuse them with many different visualization modes with too much detailed data. Also, interactive elements that allow users to get a closer look at particular data or the ability to capture specific notes are of great importance. Many dashboard templates are built within responsive web design frameworks that make it easy to customize your dashboard design. A good number of dashboard templates have also been created with the help of CSS Grid, but there are certainly more that are created within responsive web design frameworks. Free dashboard templates that are easily accessible and high quality are mostly created 
within the Bootstrap framework, while free templates within other frameworks and those created with the help of CSS Grid languages are somewhat more difficult to come by.

On the chosen samples of dashboards, according to the given criteria the best results were achieved by Material Dashboard and Stag Admin, however the others are not far behind. The results within the given criteria would nevertheless emphasize Material Design Admin Template and Stag Admin because they were not evaluated by any criterion with a score less than 4 . It can be concluded that in a given sample, according to the given evaluation criteria, Stag Admin represents a great choice when selecting a dashboard template. The gathered results are general, without taking in consideration conditions of a particular sector of industry, business world, institutions of social importance or specificity of any domain. Based on the gathered results, it is possible to test observed dashboard pattern and to confirm or refute the values presented in this paper.

\section{REFERENCE}

[1] S. Smith, Veronica. Data Dashboard as Evaluation and Research Communication Tool URL: https://doi.org/10.1002/ev.20072 (2020-01-18)

[2] Pilon, Annie. What is responsive web design URL:https://smallbiztrends.com/2013/05/what-isresponsive-web-design.html (2020-01-12)

[3] Wright, Nicholas. What is responzive web design why do you need it URL: https://www.upwork.com/hiring/mobile/what-is responsive-webdesign/ (2020-01-12)

[4] M. Rizwan Pasha, Responsive web design: present and future, MS (CS) 1-

A, Enrolment Number: 01-243151-006, 2015.

[5] Marcotte, Ethan. Responsive Web Design. URL: http://alistapart.com/article/responsive-web-design/ (2020-01-12)

[6] Introduction to RWD frameworks. URL: https://hub.packtpub.com/introduction-rwd-frameworks/ (2020-01-12)

[7] ZURB Foundation: About. URL: https://foundation.zurb.com/showcase/about (2020-01-12)

[8] Bootstrap: About. URL: https://getbootstrap.com/docs/4.3/about/overview/ (2020-01-12)

[9] Materialize: About. URL: https://materializecss.com/about.html (2020-01-12)

[10] Gerchev, Ivaylo. Introducing: Semantic UI Component Library. URL: https://www.sitepoint.com/introducing-semantic-uicomponent-library/

[11] A complete guide to Grid: Introduction. URL: https://csstricks. com/snippets/css/complete-guide-grid/ grid-introduction (2020-01-12)

[12] CSS Grid Layout Module Level 1. URL: https://www.w3.org/TR/css-grid-1/ (2020-01-12)

[13] A complete guide to Grid: Basics and Browser Support. URL: https://csstricks.com/snippets/css/complete-guidegrid/ grid-browser-support (2020-01-12)

[14] What is a data dashboard $\square$ URL: https://www.klipfolio.com/resources/articles/what-is-datadashboard (2020-01-12)

[15] Ilan Hertz, Dashboard design best practices - 4 key principles. URL:https://www.sisense.com/blog/4-designprinciples-creating-betterdashboards/

(2020-01-12)

[16] 5 zlatnih pravila za stvaranje efikasnog dashboard-a. URL:

https://oxidian.hr/5-zlatnih-pravila-za-stvaranjeefikasnog-dashboard-a/

(2020-01-12)

[17] Blitz, Shelby. 14 Useful Ways to Visualize Your Data (with Examples).URL: https://www.sisense.com/blog/10useful-ways-visualize-dataexamples/ (2020-01-12)

[18] 5 zlatnih pravila za stvaranje efikasnog dashboard-a. URL:

https://oxidian.hr/5-zlatnih-pravila-za-stvaranjeefikasnog-dashboard-a/

(2020-01-12)

[19] Noor Nashriq Ramly, Fazli Mat Nor, Nurul Haszeli Ahmad, Mohd Haris

Aziz. International Journal of Information and Education Technology:

Comparative Analysis on Data Visualization for Operations Dashboard,

Vol. 2, No.

[20] How Search algorithms work. URL:

https://www.google.com/search/howsearchworks/algorith $\mathrm{ms} /(2020-01-22)$ 